\title{
The Caenorhabditis elegans interneuron ALA is (also) a high-threshold mechanosensor
}

\author{
Jarred Sanders ${ }^{1}$, Stanislav Nagy ${ }^{2}$, Graham Fetterman ${ }^{2}$, Charles Wright ${ }^{2}$, Millet Treinin ${ }^{4}$ and David Biron $2,3^{*}$
}

\begin{abstract}
Background: To survive dynamic environments, it is essential for all animals to appropriately modulate their behavior in response to various stimulus intensities. For instance, the nematode Caenorhabditis elegans suppresses the rate of egg-laying in response to intense mechanical stimuli, in a manner dependent on the mechanosensory neurons FLP and PVD. We have found that the unilaterally placed single interneuron ALA acted as a high-threshold mechanosensor, and that it was required for this protective behavioral response.

Results: ALA was required for the inhibition of egg-laying in response to a strong (picking-like) mechanical stimulus, characteristic of routine handling of the animals. Moreover, ALA did not respond physiologically to less intense touch stimuli, but exhibited distinct physiological responses to anterior and posterior picking-like touch, suggesting that it could distinguish between spatially separated stimuli. These responses required neither neurotransmitter nor neuropeptide release from potential upstream neurons. In contrast, the long, bilaterally symmetric processes of ALA itself were required for producing its physiological responses; when they were severed, responses to stimuli administered between the cut and the cell body were unaffected, while responses to stimuli administered posterior to the cut were abolished.

Conclusion: $C$. elegans neurons are typically classified into three major groups: sensory neurons with specialized sensory dendrites, interneurons, and motoneurons with neuromuscular junctions. Our findings suggest that ALA can autonomously sense intense touch and is thus a dual-function neuron, i.e., an interneuron as well as a novel high-threshold mechanosensor.
\end{abstract}

Keywords: Mechanosensation, Physiology, Neuroethology

\section{Background}

To survive in dynamic or harsh environments, all animals must appropriately modulate their responses to various stimulus intensities. For instance, noxious stimuli are detected by nociceptors, an important class of high-threshold sensory neurons. These, in turn, lead to downstream immediate avoidance responses and enduring self-protective responses that are distinct from responses to milder stimuli [1-5]. Neurons similar to mammalian polymodal nociceptors in both function and molecular determinants have been found across the animal kingdom [6-8]. In the nematode Caenorhabditis

\footnotetext{
* Correspondence: david.biron@gmail.com

${ }^{2}$ The Institute for Biophysical Dynamics, The University of Chicago, Chicago, IL 60637, USA

${ }^{3}$ Department of Physics and the James Franck Institute, The University of Chicago, Chicago, IL 60637, USA

Full list of author information is available at the end of the article
}

elegans, the neuron types PVD and FLP have been shown to share many similarities with mammalian and Drosophila nociceptors [3,9-13], including a conservation of molecular mechanisms underlying the responses to noxious stimuli $[5,6,8,14-19]$.

C. elegans neurons are typically classified into three major groups: sensory neurons with specialized sensory dendrites, interneurons, and motoneurons with neuromuscular junctions. However, these groups are not strictly mutually exclusive. For instance, the DVA interneuron was found to be a stretch-sensitive sensory neuron [20]. ALA is a unilaterally-placed single interneuron (Figure 1A). It has a pair of bilaterally-symmetric processes that branch from the soma, and proceed along the left and right sides of the body to the tail region, adjacent to the excretory canals. A third, short process is sent from the soma to the dorsal cord [21]. ALA has been shown to be involved in reducing the velocity of animals, as well as their rate of pharyngeal

\section{Biomed Central}



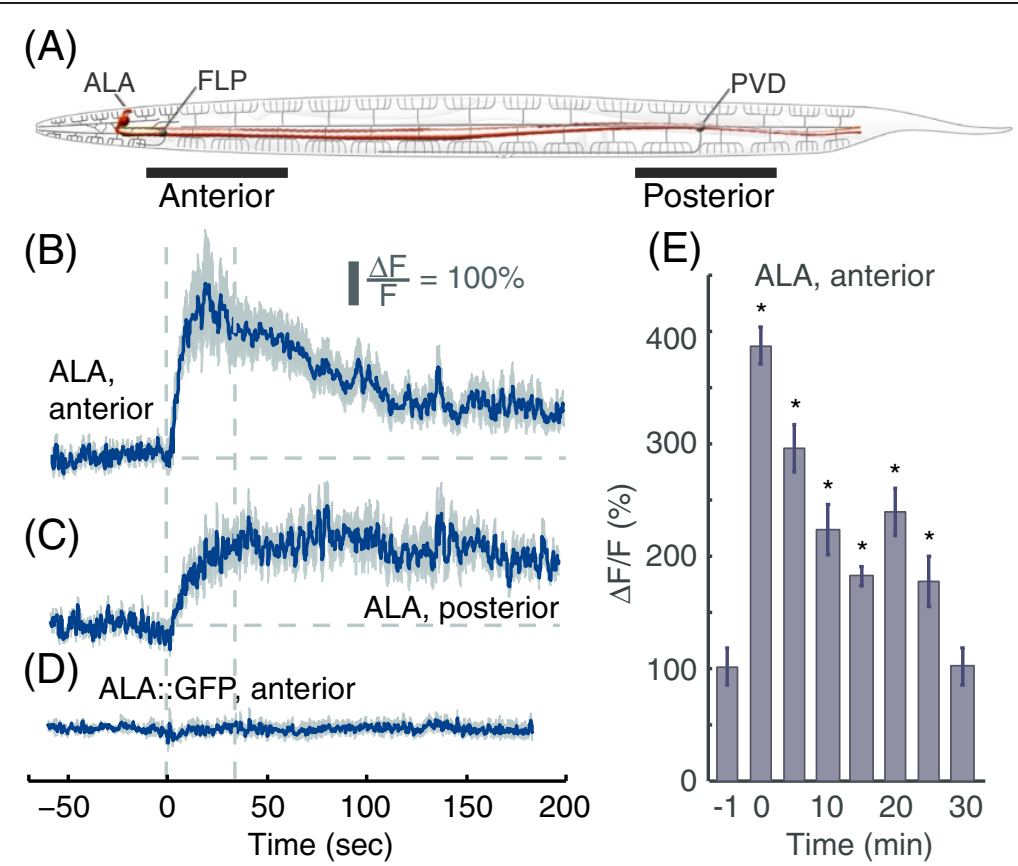

Figure 1 ALA responds to anterior and posterior picking-touch stimuli. (A) a schematic drawing of ALA (red), and the proprioceptor neurons PVD and FLP (grey). The locations where anterior and posterior mechanical stimuli were applied are denoted with black bars (schematic adapted from wormatlas.org). GCaMP3 fluorescence levels in ALA before and after administering anterior (B) or posterior (C) picking-touch stimuli at $t=0$ with a platinum wire pick. The scale bar represents a 100\% deviation from the mean baseline fluorescence. Mean \pm s.e.m, $N=9-12$ animals. (D) GFP fluorescence levels under the same conditions as in (B-C). Mean \pm s.e.m, $\mathrm{N}=10$ animals. Dashed lines in panels (B-D) are provided as a guide to the eye. (E) The mean GCaMP fluorescence levels during $20 \mathrm{sec}$ intervals, recorded 1 minute prior to administering an anterior stimulus, immediately after the stimulus, and every 5 minutes thereafter. Mean \pm s.e.m, $N=10$ animals. Asterisks denote a significant difference from the baseline mean fluorescence $(p<0.05)$.

pumping in an epidermal growth factor-dependent manner [22]. It has also been reported to decrease locomotion in a manner subject to regulation by the CEPsh sheath cells [23].

Here we show that ALA acted as a high-threshold mechanosensor, and that it played a role in a previously described response to intense mechanical stimuli [8]. ALA exhibited physiological responses to both anterior and posterior stimuli, and it was required for the inhibition of egg-laying in response to picking-touch (see Methods). The physiological responses of ALA to anterior and posterior touch were distinct, suggesting that it could distinguish between spatially separated stimuli. In addition, these responses did not require neurotransmitter or neuropeptide release from upstream neurons. However, the bilaterally symmetric processes of ALA itself were required for generating its physiological responses. These results suggest that ALA can autonomously sense picking-touch, but not lower intensity touch stimuli, and is thus a highthreshold mechanosensor.

\section{Results}

The ALA neuron responded to both anterior and posterior picking-touch stimuli

After serendipitously observing physiological responses to picking-touch (see Methods) in ALA neurons we sought to characterize these responses. In order to assay the physiological responses of ALA, we expressed the genetically encoded calcium indicator GCaMP3 [24] under the control of the ALA-specific ver-3 promoter [22,25-34]. Touch stimuli were applied to either the anterior or the posterior region of the animal, and the resulting fluorescence intensity of the cell soma was recorded. We did not observe a response either to gentle-touch or to harshtouch in ALA neurons (see Methods). Therefore, ALA was not found to be a low-threshold mechanosensor. In contrast, both anterior and posterior picking-touch evoked calcium transients in ALA (Figure 1). In the case of anterior stimuli, GCaMP fluorescence exhibited a $10 \mathrm{sec}$ increase to $400 \%$ of the baseline followed by a decline to $200 \%$ of the baseline after $100 \mathrm{sec}$ (Figure 1B). Posterior stimuli resulted in a gradual $50 \mathrm{sec}$ increase in fluorescence to $300 \%$ of the baseline (Figure 1C). In both cases, GCaMP3 fluorescence did not return to baseline within the 5 minutes of the measurement but sustained similar levels of $200 \%$ of the baseline (see also Figure 2). To test how long ALA remained active while minimizing photobleaching, we recorded it for 20 sec every 5 minutes. We found that 30 minutes after an anterior stimulus had been given, GCaMP3 fluorescence was not significantly different than its pre-stimulus baseline (Figure 1E). 
To control for motion artifacts, the fluorescence of Pver-3::GFP was assayed for anterior picking-touch under identical conditions and did not deviate from its baseline level (Figure 1D). A negative control (Figure 3A) was provided by the absence of detectable physiological responses in the dopaminergic CEPD/L head neurons [35] despite the fact that the ALM neurons were previously shown

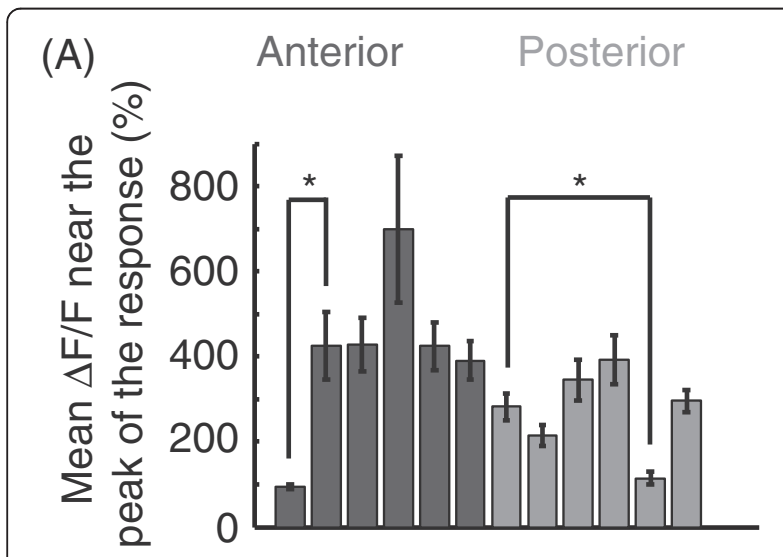

(B)

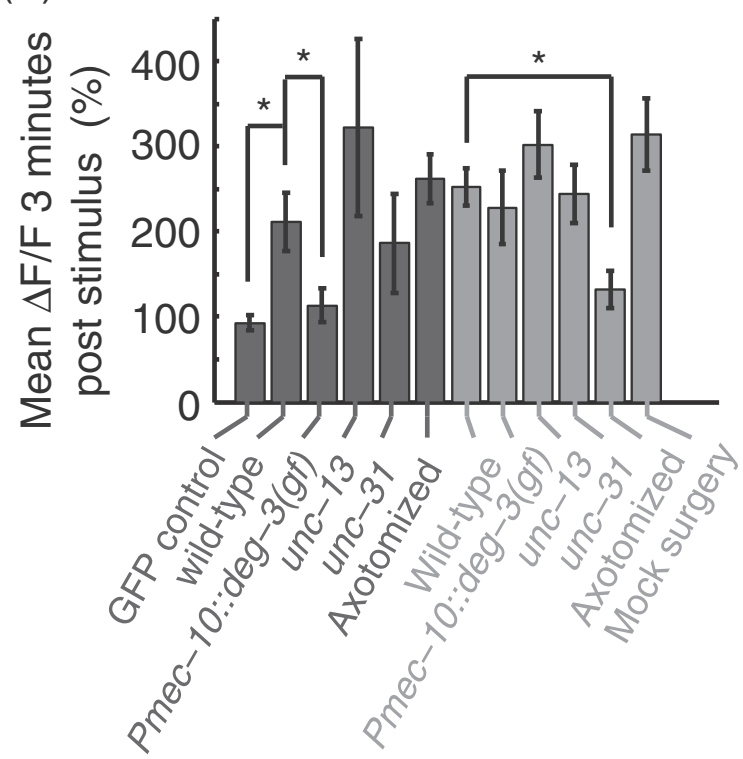

Figure 2 A comparison of ALA physiological responses to picking-touch. (A) The mean GCaMP fluorescence during the 20 seconds surrounding peak activity, $t=5-25 \mathrm{sec}$ and $t=40-60 \mathrm{sec}$ post-stimulus for anterior and posterior stimuli, respectively. Asterisks denote that the measurements of GFP (control) fluorescence and the post-surgery responses to posterior stimuli were significantly different from the corresponding wild-type responses $(p<0.05)$. (B) The mean GCaMP fluorescence at $\mathrm{t}=160-180 \mathrm{sec}$. Asterisks denote that the measurements of GFP (control) fluorescence, the responses in Pmec-10:: deg-3(4662) animals, and the post-surgery responses to posterior stimuli were both significantly different from the corresponding wild-type responses $(p<0.05)$. All mean fluorescence levels were calculated from the datasets shown in Figures 1, 5, 7, and 8.

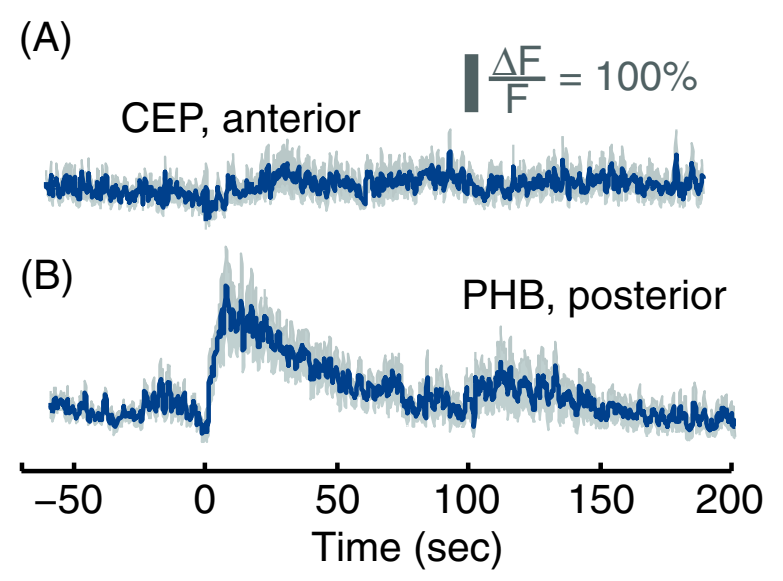

Figure 3 CEP neurons did not exhibit a detectable response to anterior picking-touch, and $\mathrm{PHB}$ neurons responded to posterior picking-touch. GCaMP3 fluorescence levels in CEPDL/R neurons before and after administering anterior stimulus (A) and in PHB neurons before and after administering a posterior stimulus (B) at $\mathrm{t}=0$ with a platinum wire pick. The scale bar represents a 100\% deviation from the mean baseline fluorescence. Mean \pm s.e.m, $\mathrm{N}=10$ animals.

to indirectly activate $\mathrm{CEP}$ in response to an appropriate anterior body touch [36]. As a positive control, we expressed GCaMP3 in the phasmid chemosensory PHB neurons. Although the physiological responses of PHB neurons to harsh touch have not been previously reported, they were implicated in posterior harsh touch in a laser ablation study [32]. In response to posterior picking-touch, we observed a 3-fold increase in GCaMP fluorescence in PHB, which returned to baseline level after $100 \mathrm{sec}$ (Figure 3B). These results suggest that ALA either received input from an upstream highthreshold mechanosensor or sensed picking-touch autonomously. In addition, the differences in the temporal dynamics of the responses to anterior and posterior picking-touch suggested that the neuron sensing the stimulus was able to discriminate between different spatial positions of stimuli along the body of the animal.

A mutation that impairs ALA differentiation abolished the reduced egg-laying response to picking-touch

The commonly performed act of transferring a single animal to a new plate using a platinum wire pick inadvertently delivers an intense mechanical stimulus to the animal and evokes a characteristic behavioral response. It was previously shown that this mechanical stimulus leads to PVD- and FLP-mediated egg-laying inhibition lasting 30 minutes [8]. The same procedure was also shown to affect locomotion on a timescale of several minutes [37]. In addition, posterior gentle touch was shown to lead to increased intervals between calcium 
transients of the HSN egg-laying neurons [38]. We thus asked whether ALA might have a role in mediating these behavioral responses. To address this question, we compared the suppression of egg-laying in wild-type animals to animals mutant for ceh-17, a gene encoding a pairedlike homeodomain transcription factor.

CEH-17 is expressed in ALA and the cholinergic SIA head neurons and is involved in longitudinal axonal navigation and ALA differentiation [22,39]. Specifically, $\mathrm{CEH}-17$ was shown to regulate the expression of the tyrosine phosphatase-like receptor gene ida-1, which is required for ALA neuropeptide release, and ceh-17 mutants exhibited shortened ALA processes [38,40]. Since the ALA neuron has two bilaterally symmetrical processes that extend from the cell body to the tail of the animal [21], we assayed responses to anterior and posterior stimuli separately. In agreement with previous observation, in our hands both anterior and posterior picking-touch led to a 35\% reduction in the number of eggs that were laid by wild-type animals during a 3-hour period. In contrast, in ceh-17(np1) mutants the inhibition of egg-laying in response to both anterior and posterior stimuli was completely abolished (Figure 4). In agreement with previous findings [39], we did not observe differences between the locomotive responses of wild-type animals and ceh-17 mutants to picking-touch (data not shown).

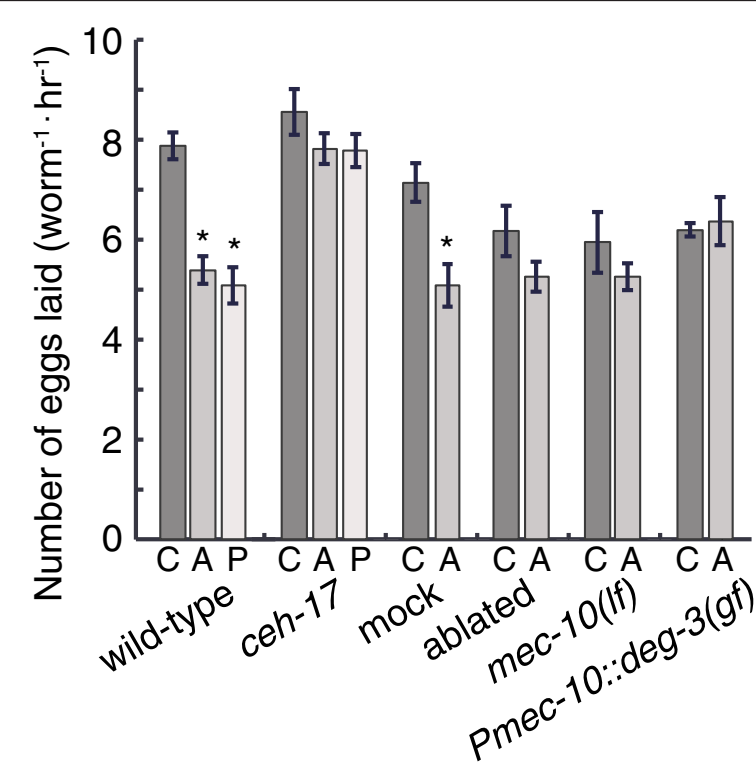

Figure 4 ALA is required for the suppression of the egg-laying response to picking-touch. The number of eggs laid per animal, per hour, in animals exposed to anterior or posterior picking-touch stimuli (" $A$ " and " $P$ " respectively) and in control animals $\left({ }^{\prime \prime} C\right.$ "). Mean \pm s.e.m, $\mathrm{N}=4-13$ assay plates (see Methods). Asterisks denote statistically significant comparisons $(p<0.05)$.

\section{Laser ablation of ALA abolished the reduced egg-laying response to picking-touch}

To specifically test whether ALA was required for the egglaying inhibition response to picking-touch, we assayed animals in which the ALA neuron was laser ablated during the L4 larval stage. As opposed to the mock-ablated control animals, ALA-ablated animals did not exhibit suppression of the egg-laying response (Figure 4). However, the egg-laying rate of unperturbed post-surgery animals was variable, with a lower mean (although not significantly: $p$ $=0.114$ by one-way ANOVA) than the corresponding rate in mock-ablated controls. This variability may be indicative of unspecific damage of the surgery that could not be avoided. We obtained similar results (Figure 4) for two strains in which behavioral responses to harsh touch were abolished [6,8]: mutants for the mec-10 gene, encoding an amiloride-sensitive sodium channel protein of the DEG/ $\mathrm{ENaC}$ family that is required for $C$. elegans touch sensation [3,9,11-13], and animals where a degeneration-causing, constitutively active nicotinic acetylcholine receptor (nAChR) channel subunit, deg-3(u662), was expressed under a Pmec-10 promoter $[8,14,16]$, driving expression in and degeneration of PVD, FLP and the six touch receptor neurons [1,11]. The suppression of egg-laying was not significant in mec-10(tm1552) mutants ( $\mathrm{p}=0.193$ by oneway ANOVA) or Pmec-10::deg-3(u662) animals ( $\mathrm{p}=0.732$ by one-way ANOVA) [2,3,6,32,41]. Taken together with the responses of ceh-17 mutants, our results suggest that ALA is required for the inhibition of egg-laying in response to picking-touch, but not for immediate avoidance responses.

The harsh-touch sensory neurons were not required for the physiological response of ALA to picking-touch

The interneuron ALA has not been previously reported to act as a mechanosensory neuron, but the effects on egg-laying of ablating it were similar to those of ablating known mechanosensors. Moreover, although synapses between ALA and PVD have not been previously found, the elongated processes of ALA and the primary dendrites of PVD are in close proximity [3,4,21]. It was thus possible that ALA transduced signals from known mechanosensory neurons, so we asked whether the physiological responses that we observed were dependent on input from them. To test this, we crossed our Pver-3::GCaMP3 reporter into transgenic animals expressing the degeneration-causing nAChR channel subunit, deg-3(u662), in PVD, FLP and the six harsh touch neurons $[7,14,16]$. In agreement with previous results $[8,10,11]$, these animals failed to respond to standard harsh touch stimuli (data not shown). In addition, the physiological responses of their ALA neurons to posterior picking-touch were unaffected by the genetic ablation (Figures 5B and 2). However, in response to anterior picking-touch, GCaMP fluorescence in ALA returned 


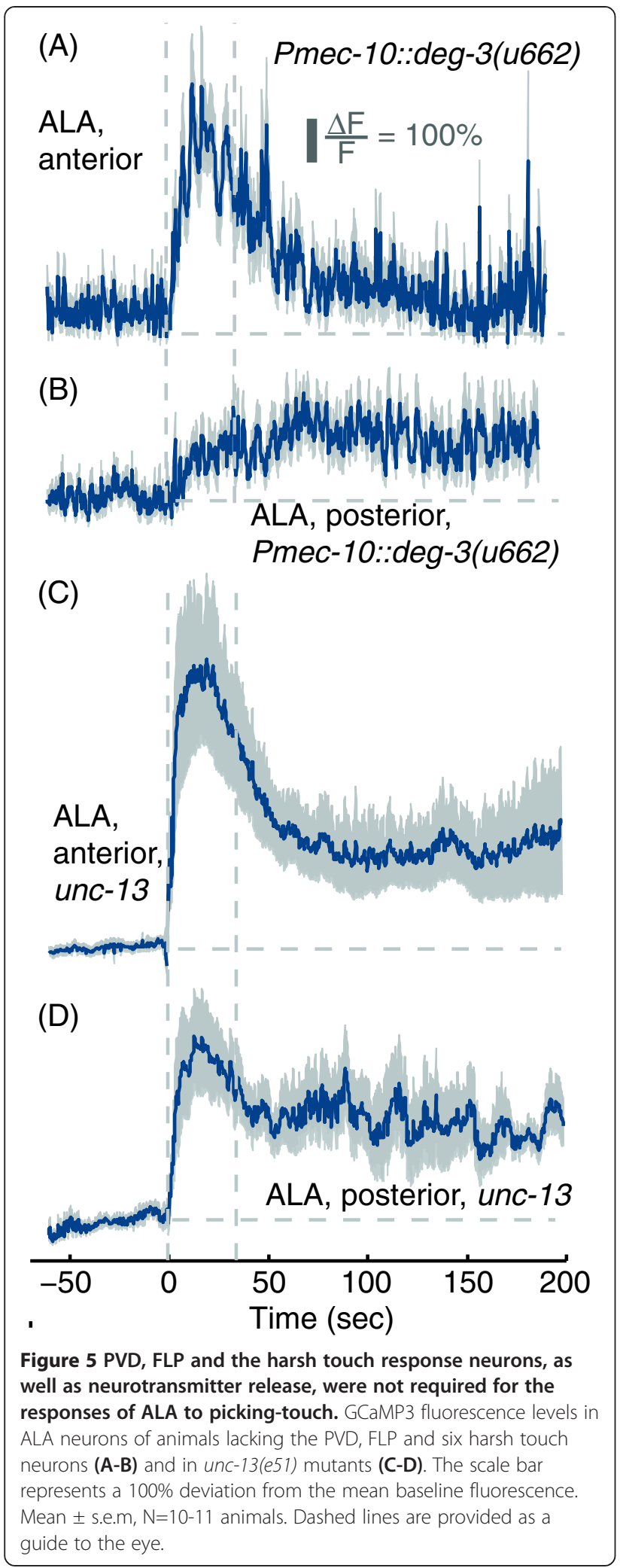

to its baseline level after 2 minutes in these transgenics (Figure 5A and 2), in contrast to the wild-type prolonged response to the same stimulus. We concluded that the responses of ALA did not require the function of PVD, FLP and the six touch receptor neurons, although a subgroup of these neurons may have a role in sustaining the responses to anterior stimuli.

\section{The physiological response of ALA to picking-touch did not require neurotransmitter release}

Although physiological responses in ALA did not require known mechanosensory neurons, it was still possible that the responses were dependent on input from other presynaptic partners of ALA. To answer this question we crossed our Pver-3:GCaMP3 reporter into an unc-13(e51) mutant background, where synaptic vesicle exocytosis is essentially eliminated [6,8,15,17-19,42,43]. Anterior and posterior picking-touch evoked rapid and long-lasting calcium transients in the ALA neurons of unc-13(e51) mutants (Figures 5C-D and 2). The peaks of unc-13(e51) responses to anterior stimuli were highly variable, with a coefficient of variance of 0.78 (Figure 2A), but were not significantly different from wild-type $(\mathrm{p}=0.103$ by oneway ANOVA). In contrast to wild-type, the rise time of the responses to posterior stimuli was rapid $(5 \mathrm{sec})$ in the mutant animals, and their dynamics were similar to those of responses to anterior stimuli. These results suggested that synaptic input from neurotransmitter release was not necessary for producing the responses of ALA to pickingtouch. However, such input may contribute to more subtle aspects of regulating these responses (similar to the case of the Pmec-10::deg-3(u662) transgenic background) such as the initial dampening of responses to posterior stimuli.

\section{Presynaptic partners of ALA responded to anterior picking-touch}

Since blocking neurotransmitter release modulated the kinetics of the responses of ALA, we asked whether its presynaptic partners responded to picking-touch. Specifically, we focused on two presynaptic sensory neurons: $\mathrm{ADE}$, previously implicated in anterior touch responses by laser ablation experiments [32,36,44-46], and ADL, a polymodal nociceptive neuron [47-50]. We expressed GCaMP3 in ADE (and all of the dopaminergic neurons) and ADL using the Pdat-1 [21,51] and Psrh-220 [22,52] promoters, respectively. ADE neurons exhibited a slowly rising response to anterior but not posterior pickingtouch, which peaked after $40 \mathrm{sec}$ and returned to baseline after 5 min (Figure 6A-B). However, abolishing vesicle exocytosis in all dopaminergic neurons by driving expression of the tetanus toxin light-chain with the dat-1 promoter $[23,53,54]$ did not significantly alter the responses of ALA to picking-touch (Figure 6C-D). Consistent with previous reports [8,50], ADL neurons responded to the onset of blue light. However, this response decayed after 4 minutes of continuous illumination such that the animal could be assayed approximately 10 minutes after initially 


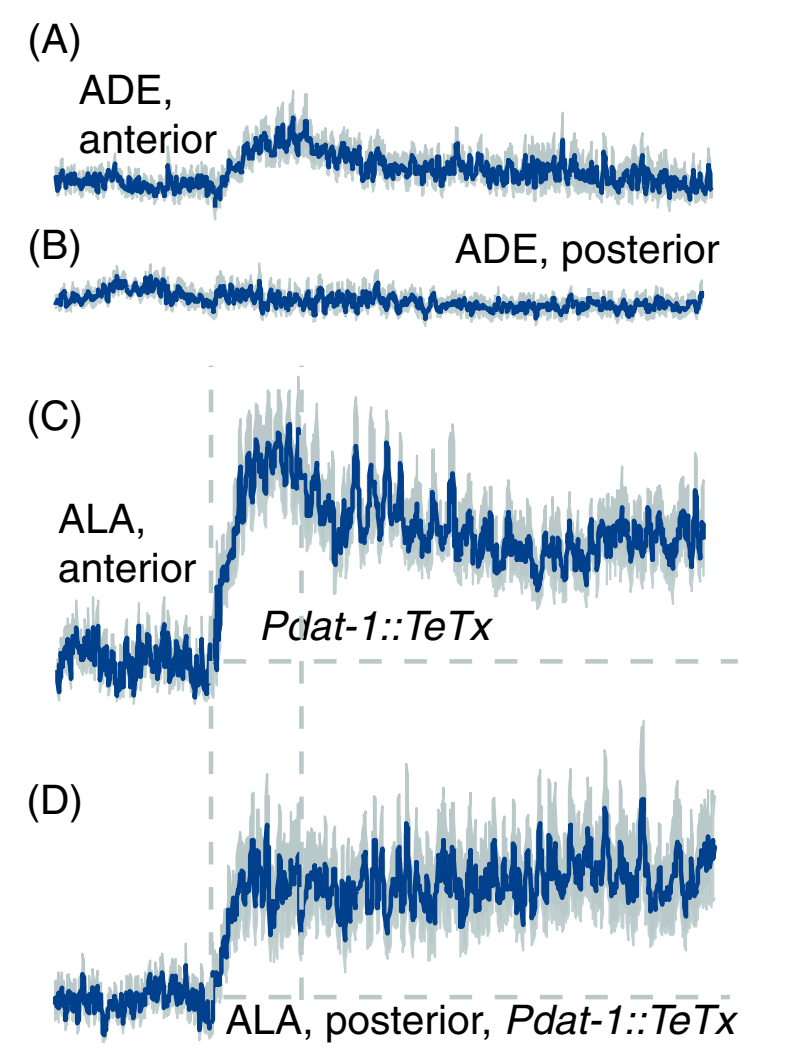

(E)

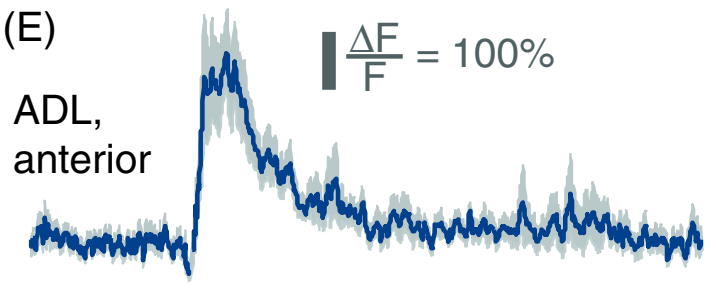

$(\mathrm{F})$

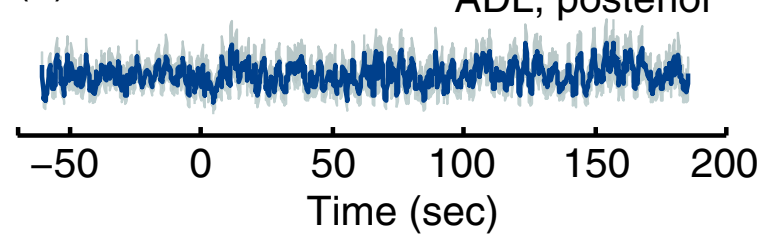

Figure 6 The sensory neurons ADE and ADL, both presynaptic partners of $A L A$, responded to anterior but not to posterior picking-touch. GCaMP3 fluorescence levels in ADE neurons (A-B), ALA neurons in animals expressing the tetanus toxin light chain in their dopaminergic neurons (C-D) and in ADL neurons (E-F). In all cases responses to both anterior and posterior stimuli are shown. Mean \pm s.e.m, $N=10$ animals. Dashed lines in panels (C-D) are provided as a guide to the eye.

being exposed to light. ADL neurons also responded to anterior, but not posterior picking-touch, and their response decayed to baseline after $70 \mathrm{sec}$ (Figure 6E-F). Taken together with the responses observed in $u n c-13$ mutants, these results indicated that synaptic input from
$\mathrm{ADE}$ and ADL did not contribute to the responses of ALA to picking-touch.

ADL, a presynaptic partner of ALA, has been shown to be a chemosensory neuron that plays a role in avoidance behavior in the presence of volatile repellents such as octanol $[48,55]$. Ablating ADL has been shown to increase the latency of responses to octanol off food, but not on food $[8,56]$. We thus sought to test whether ALA might be mediating octanol avoidance in addition to responses to picking-touch. To answer this question, we dipped an eyebrow hair in 30\% octanol and presented it in front of the nose of Pver-3::GCaMP3 animals. We observed reversals characteristic of the avoidance response of C. elegans in both wild-type animals and ceh-17 mutants, with latencies of $4 \mathrm{sec}$ in both genetic backgrounds, both on and off food (data not shown). Consistent with the behavioral assay, the repellent did not evoke detectable calcium transients in the ALA neuron (data not shown). Taken together, these results suggested that ALA did not mediate octanol avoidance.

The physiological responses of ALA to picking-touch were independent of neuropeptide release

Although input from neurotransmitter release of presynaptic partners of ALA was not required for producing its physiological responses to picking-touch, it could be the case that peptidergic signaling from an unknown mechanosensory neuron was required. The $u n c-31$ gene encodes an ortholog of the mammalian CAPS protein and is required for dense-core vesicle but not synaptic vesicle exocytosis of neuropeptides [32,57-59]. Thus, we crossed our Pver-3::GCaMP3 reporter into an unc-31(e169) mutant background, where dense-core vesicle exocytosis is practically eliminated. Anterior picking-touch evoked wild-type-like responses in the ALA neurons of unc-31 (e169) mutants, while posterior touch evoked responses that appeared slightly enhanced during the first $50 \mathrm{sec}$ post-stimulus, but not significantly so (Figures 7 and 2: $\mathrm{p}$ $=0.998$ and $\mathrm{p}=0.162$ by one-way ANOVA for anterior and posterior stimuli, respectively). These results suggested that neuropeptide release was not required for evoking or sustaining the responses of ALA to pickingtouch.

The elongated processes of the ALA neuron are required for its physiological response to picking-touch

We reasoned that if ALA was autonomously sensing picking-touch, then it would likely require its long, bilaterally symmetric processes to do so. If this is the case, severing the elongated processes between the nerve ring and the vulva should abolish ALA responses, at least to stimuli that are posterior to the cut. We used a femtosecond pulsed laser to sever ALA processes [32,57,60-63] in wild-type L4 larvae expressing our reporter, Pver-3:: 


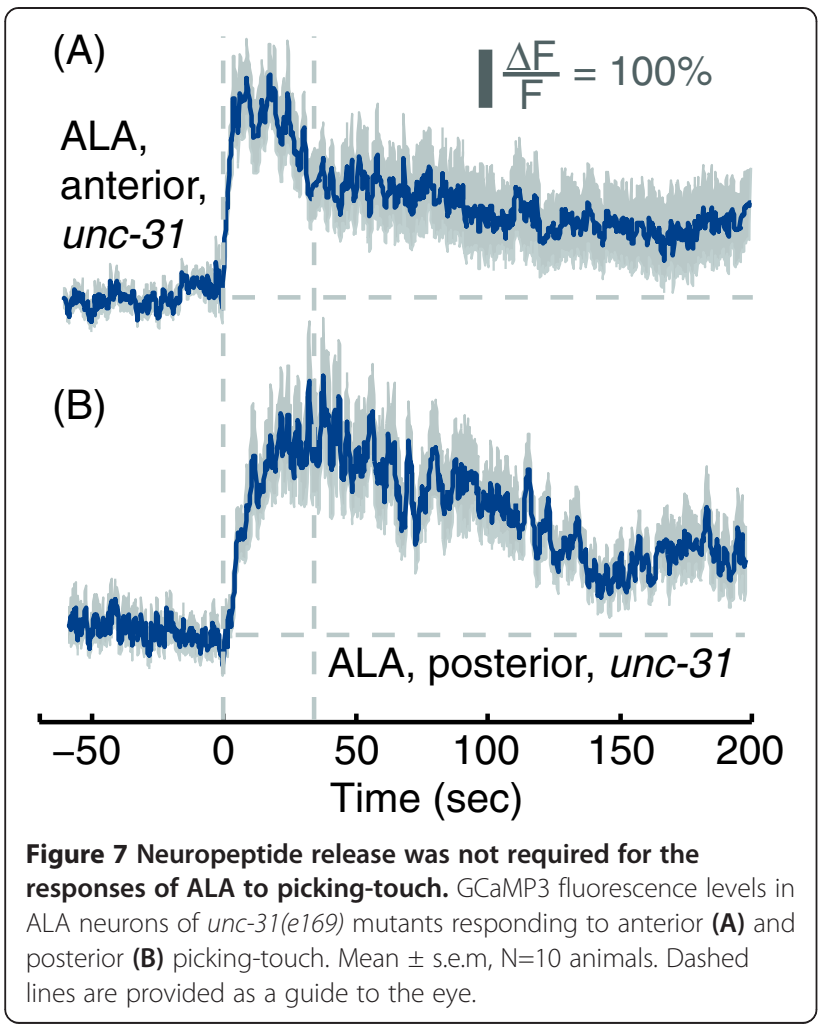

GCaMP3, and assayed them as young adults 24 hours post-surgery (Figure 8A). We found that the physiological responses of ALA to posterior picking-touch downstream from the cut were completely abolished in axotomized animals and unaffected in the mock-axotomy controls (Figures $8 \mathrm{~B}-\mathrm{C}$ and 2). In contrast, ALA responses to anterior picking-touch, upstream from the cut, were unaffected by the surgery (Figures $8 \mathrm{D}$ and 2). The wild-type like responses to anterior stimuli suggested that the part of the cell upstream from the cut, including the soma, was not damaged by the surgery - an internal control that is typically difficult to obtain. These results supported the conclusion that ALA autonomously sensed pickingtouch stimuli and that its long processes were required for this function. In addition, they suggested that ALA could discriminate between different spatial positions of stimuli along the body.

\section{Discussion and conclusions}

In this study we show that the ALA interneuron of the nematode C. elegans exhibits physiological responses to picking-touch and that it is required for a stereotypical response to this stimulus, a suppression of egg-laying $[8,32,38]$. The physiological responses of ALA did not require input from presynaptic release of neurotransmitters, nor did they require neuropeptide release. When the elongated, bilaterally symmetric processes of ALA were axotomized, the physiological responses to stimuli

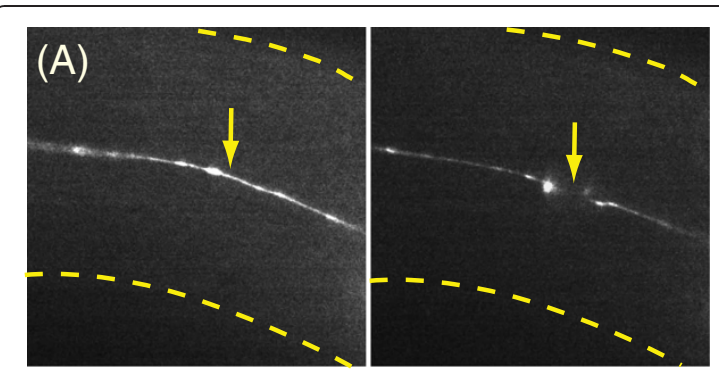

(B)

ALA, posterior, axotomized

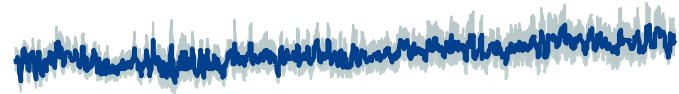

(C)

$\frac{\Delta \mathrm{F}}{\mathrm{F}}=100 \%$
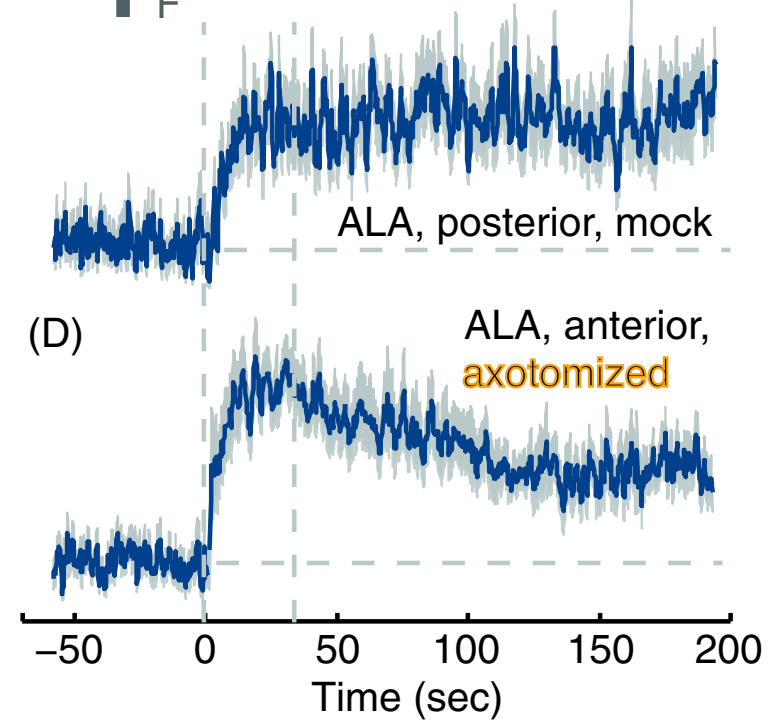

Figure 8 Severing both of the elongated processes of ALA abolished its responses to picking-touch stimuli that were positioned posterior to the cut. (A) A representative image of a single ALA process expressing GCaMP3 before (left) and after (right) it was severed. (B-C) GCaMP3 fluorescence levels in ALA neurons before and after a picking-touch stimulus was administered posterior to the cut in operated animals (B) and in mock-operated controls (C). Mean \pm s.e.m, $N_{\text {operated }}=8$ and $N_{\text {mock }}=10$ animals. (D) Same as (B), but for stimuli that were administered anterior to the cut, i.e., between the cut and the ALA cell body. Mean \pm s.e.m, $N_{\text {operated }}=11$ animals. Dashed lines in panels (C-D) are provided as a guide to the eye.

that were on the anterior side (upstream) of the cut persisted, while the responses to stimuli on the posterior side (downstream) of the cut were eliminated. ALA is known to form several electrical synapses, all of which are located in the anterior sections of its processes, i.e., in vicinity of the cell soma $[21,64]$. As a result, our axotomy experiments rule out the possibility that input from the known gap junctions of ALA was capable of producing the 
observed responses. Taken together, our findings suggest that ALA can sense picking-touch stimuli autonomously, i.e., that it is a high-threshold mechanosensor.

The ALA neuron could discriminate between spatially separated stimuli along the body of the animal. Three observations are consistent with a model in which (as of yet unknown) molecular sensors are distributed along the processes of ALA: (1) the temporal dynamics of the responses to anterior and posterior stimuli were distinct, (2) neurotransmitter and neuropeptide release affected anterior and posterior responses differently, and, most importantly, (3) when the elongated processes of ALA were severed the responses to stimuli applied posterior but not anterior to the cut were abolished. Measuring the receptive field map of ALA using more accurately localized stimuli could provide insight into the spatial differentiation of this mechanosensor.

The physiological responses that we observed in ALA were unusually prolonged as compared to typical responses of $C$. elegans sensory neurons [36,61,62,65-70]. Application of menthol, a noxious stimulus, can produce similarly prolonged calcium transients in mammalian nociceptors [71]. However, axonal injury in mammalian and invertebrate preparation can also produce longlasting calcium responses [72,73]. Could the physiological and behavioral responses that we observed result from a compression or strain injury inflicted on ALA by pickingtouch? Several considerations suggest that this is unlikely: (1) picking was used to transfer "control" animals to assay plates, as well as for their maintenance, such that a potential injury in ALA would be required to heal on the timescale of an hour in order to allow for the higher egg-laying rate in the absence of the recurring stimulus, (2) ALA is not at all unique in being located peripherally or having elongated processes; similar anatomical features are characteristic of many touch receptor, proprioceptor and motor neurons in C. elegans [21], (3) egg-laying was not suppressed in response to anterior picking-touch in ceh17 mutants, where the anterior half of the ALA process was typically present; moreover, the developmental defect in ALA neurons in ceh-17 mutants did not suppress egglaying, and (4) in C. elegans, axotomy induced calcium dynamics in the soma of the touch receptor neuron ALM exhibited a strong dependence on the lesion distance, effectively vanishing when the axon was injured merely $40 \mu \mathrm{m}$ from the soma [73]. Thus, explaining the reported responses as a result of injury would require multiple unsupported assumptions regarding the unique nature of ALA, such as an unexplained enhanced vulnaribility to compression, an ability to heal rapidly, an effect on egg-laying in response to injury that is distinct from a non-specific loss of function, and post-injury calcium dynamics dissimilar from those of a C. elegans touch receptor neuron.
Our findings associate the responses of ALA to pickingtouch with the enduring inhibition of egg-laying, but not with an immediate avoidance response. Since enduring behavioral responses have been shown to depend on neuropeptides in previous studies $[8,35]$, we hypothesize that this could also be the case here. Based on its anatomical features, it has been suggested that ALA may be a neurosecretory neuron $[21,36,74]$. It has been shown that ALA is required for the regulation by epidermal growth factor (EGF) signaling of feeding and locomotion patterns, and that feeding defects caused by overexpression of the EGFlike peptide LIN-3 are suppressed by a mutation in the gene encoding UNC-31/CAPS [22,32]. In addition, the tyrosine phosphatase-like receptor gene ida-1 was shown to be expressed in ALA $[8,40,75]$. IDA-1 was demonstrated to be important for dense-core vesicle cargo release; it acts genetically in the signaling pathway of unc-31 (encoding the UNC-31/CAPS protein) suggesting that it has a role in the trafficking of dense core vesicles and/or in their cargo release $[37,76]$. Importantly, $\mathrm{CEH}-17$ was shown to regulate the expression of ida-1 in ALA [38,40], such that neuropeptide release from ALA would be expected to be impaired in ceh-17 mutants. Taken together, these findings and the results presented here are consistent with the idea that ALA may use peptidergic signaling to communicate to its downstream targets.

Several C. elegans neurons, such as the proprioceptors DVA, AVG, and PVR, were originally classified as interneurons and later found to also function as sensory neurons. In addition, interneurons functioning also as sensory neurons have been described in other species, e.g. the B51 neuron in Aplysia californica [77,78]. However, this study was the first to associate the role of a mechanosensor with the interneuron ALA, thus demonstrating that it is a dualfunction neuron. The roles of neuropeptides in modulating the repertoire of enduring responses, as well as the degree to which these roles may be either evolutionarily conserved, remain to be understood.

\section{Methods}

\section{Strains}

Wild-type, transgenic, and mutant $C$. elegans strains were maintained and cultivated with OP50 bacteria according to standard protocols [55]. The following strains were used: wild-type strain N2, INV21001 N2;Ex[Pver-3::GCaMP3], INV21002 N2;Ex[Pgpc-1::GCaMP3], INV21003 N2;Ex[Psr h-220::GCaMP3], INV21004 N2;Ex[Pdat-1::GCaMP3], IN V54001 N2;Ex[Pdat-1::TeTx; Pver-3::GCaMP3], INV54002 egl-3(n150)V; Ex[Pver-3::GCaMP3], INV54003 unc-13(e51); Ex[Pver-3::GCaMP3], INV54004 unc-31(e169); Ex[Pver-3:: GCaMP3] and INV54005 N2;Is[Pmec-10::deg-3(u662)]; Ex [Pver-3::GCaMp3]. The OS5513 N2; Ex[Pver-3::GFP] and the IB16 ceh-17(np1)I strains were a gift from Menachem Katz of Shai Shaham's laboratory (Rockefeller University). 


\section{Picking-, harsh- and gentle-touch stimuli}

The routine maintenance procedure of picking animals entails applying pressure with a platinum wire covered with a thin sticky layer of bacteria, a procedure performed on the order of 10,000 times a year by a typical experimenter. Although it is difficult to obtain quantitative characterizations of hand-delivered mechanical stimuli, estimates of the pressure applied by a platinum wire pick were previously obtained using an NGM test plates placed on an analytical balance [32]. By this measure, in our hands, picking-touch was estimated to involve pressures that were 10-fold larger than those associated with the standard harsh-touch stimulus. Thus, C. elegans researchers commonly apply three distinct tiers of touch stimuli: (1) gentle touch - the weakest intensity tier, e.g., brushing the animal with an eye-lash pick, (2) harsh touch - an intermediate intensity tier, e.g., probing with a platinum wire or a glass rod, and (3) picking touch - the highest intensity tier in routine use $[32,57,60]$. In addition, picking-touch was previously shown to produce a behavioral response - a suppression of the rate of egg-laying [8]. In our assays, a pickingtouch stimulus was delivered in the same manner as in routine picking, but without the thin bacteria layer that would facilitate the lifting of the animal from the substrate. The duration of the stimulus was approximately one second. Thus applied, this common procedure was not previously reported to result in sustained tissue damage, and our data suggests that it did not injure the ALA neuron. To test responses to lower intensity mechanical stimuli, we delivered standard harsh-touch and gentle-touch stimuli using a platinum wire and an eyelash pick, respectively.

\section{Octanol avoidance assay}

Avoidance of 30\% 1-octanol was assayed as previously described $[48,56]$. In brief, an eyelash hair was attached to a Pasteur pipette, dipped in octanol, and presented in front of a forward moving animal without touching it. The amount of time it took the animal to initiate backward movement was determined by a handheld audible timer. Two well-fed animals were placed on fresh NGM plates (either with or without a lawn of bacterial food) 30 minutes prior to each assay. Each animal was tested 7-10 times, with an interval of at least 2 minutes between successive tests.

\section{Egg-laying assay}

Animals were synchronized and assayed at 70 hours posthatching unless noted otherwise. Animals were placed in triplets on a $3 \mathrm{~cm}$ diameter NGM plates at $20^{\circ} \mathrm{C} 20$ minutes prior to the beginning of the assay. Picking-touch stimuli were delivered manually to each individual animal every 20 minutes during a 3 hours period. The adults were then removed and the number of eggs per plate was counted. To simplify egg-counting, each assay plate was seeded with a small drop of OP50 bacteria at its center.

\section{Physiological imaging in freely behaving animals}

Animals were manually synchronized by transferring 10 gravid adults to fresh NGM plates and restricting the duration of egg-laying to two hours. The adults were then removed, and the embryos were grown at $20^{\circ} \mathrm{C}$ until young adulthood. One hour prior to testing, young adult animals expressing the appropriate marker were transferred to a fresh standard NGM plate $(6 \mathrm{~cm}$ in diameter) spread with a thin layer of OP50 bacteria. Picking-touch stimuli were delivered manually to freely moving animals using a platinum-iridium wire $(0.2 \mathrm{~mm}$ diameter, $99.9 \%$ purity from Alfa Aesar) attached to a glass Pasteur pipette [32]. Anterior and posterior stimuli were applied to the farthest quartiles of the animal body, respectively. A single stimulus was delivered per animal. Calcium imaging of each worm was performed at a magnification of $11.5 \times$ for $1 \mathrm{mi}$ nute prior to the stimulus and 4 minutes post-stimulus. Alternatively, in order to capture the slow decay of the signal, 20 seconds of imaging were performed every 5 minutes for 30 minutes. Images were binned $4 \times$, and captured at $5 \mathrm{~Hz}$ using a cooled CCD camera (Photometrics CoolSNAP HQ2, Tucson, AZ), an Olympus SZX16 stereomicroscope equipped with an SDF PLAPO 1XPF objective (Olympus America Inc., Center Valley, PA), and Micromanager [79]. Animals were tracked manually during the assay and image analysis was performed using custom MATLAB scripts (Mathworks, Inc. Natick, MA).

\section{Axotomy and ablations}

Ablations and axotomy were performed on L4 larvae, immobilized with $10 \mu \mathrm{M}$ levamisole on a $2 \%$ agarose pad, using a Ti:sapphire femtosecond laser, as previously described [61-63]. The transgenes Pver-3::GFP and Pver3::GCaMP3 were used to identify ALA neurons for ablations and axotomy, respectively.

\section{Competing interest}

On behalf of the authors of the manuscript, I wish to state that the authors declare no financial or non-financial competing interests (DB).

\section{Authors' contribution}

JS carried out the molecular, behavioral, and physiological studies and drafted the manuscript. GF carried out behavioral studies. SN and MT participated in the molecular work. CR participated in the data analysis. DB conceived of the study, and participated in its design and coordination and helped to draft the manuscript. All authors contributed to writing and editing of drafts, and approved the final manuscript.

\section{Acknowledgements}

Some of the strains used in this study were provided by the Caenorhabditis Genetics Center, which is funded by the NIH Office of Research Infrastructure Programs (g0072ant no. P40 OD010440). This work was supported by the NIH Training Grant 2T32GM007197-37 (JS), the Burroughs Wellcome Fund Career Award at the Scientific Interface (DB) and the Searle Scholars Program (DB). 


\section{Author details}

'Committee on Genetics, Genomics, and Systems Biology, The University of Chicago, Chicago, IL 60637, USA. ${ }^{2}$ The Institute for Biophysical Dynamics, The University of Chicago, Chicago, IL 60637, USA. ${ }^{3}$ Department of Physics and the James Franck Institute, The University of Chicago, Chicago, IL 60637, USA. ${ }^{4}$ Department of Medical Neurobiology, Institute for Medical Research Israel-Canada, Hebrew University - Hadassah Medical School, Jerusalem 91120, Israel.

\section{Received: 12 July 2013 Accepted: 6 December 2013}

Published: 17 December 2013

\section{References}

1. Bromm B, Treede RD: Withdrawal reflex, skin resistance reaction and pain ratings due to electrical stimuli in man. Pain 1980, 9:339-354.

2. Wittenburg N, Baumeister R: Thermal avoidance in Caenorhabditis elegans: an approach to the study of nociception. Proc Natl Acad Sci USA 1999, 96:10477-10482.

3. Basbaum Al, Bautista DM, Scherrer G, Julius D: Cellular and molecular mechanisms of pain. Cell 2009, 139:267-284.

4. Gold MS, Gebhart GF: Nociceptor sensitization in pain pathogenesis. Nat Med 2010, 16:1248-1257.

5. Mohammadi A, Byrne Rodgers J, Kotera I, Ryu WS: Behavioral response of Caenorhabditis elegans to localized thermal stimuli. BMC Neurosci 2013, $14: 66$

6. Chatzigeorgiou M, Yoo S, Watson JD, Lee W-H, Spencer WC, Kindt KS, Hwang SW, Miller DM, Treinin M, Driscoll M, Schafer WR: Specific roles for DEG/ENaC and TRP channels in touch and thermosensation in C. elegans nociceptors. Nat Neurosci 2010, 13:861-868.

7. Smith ESJ, Lewin GR: Nociceptors: a phylogenetic view. J Comp Physiol A Neuroethol Sens Neural Behav Physiol 2009, 195:1089-1106.

8. Albeg A, Smith CJ, Chatzigeorgiou M, Feitelson DG, Hall DH, Schafer WR, Miller DM, Treinin M: C. elegans multi-dendritic sensory neurons: morphology and function. Mol Cell Neurosci 2011, 46:308-317.

9. Way JC, Chalfie M: mec-3, a homeobox-containing gene that specifies differentiation of the touch receptor neurons in C. elegans. Cell 1988, 54:5-16.

10. Hall DH, Treinin M: How does morphology relate to function in sensory arbors? Trends Neurosci 2011, 34:443-451.

11. Huang M, Chalfie M: Gene interactions affecting mechanosensory transduction in Caenorhabditis elegans. Nature 1994, 367:467-470

12. Goodman MB, Ernstrom GG, Chelur DS, O'Hagan R, Yao CA, Chalfie M: MEC -2 regulates $C$. elegans $D E G / E N a C$ channels needed for mechanosensation. Nature 2002, 415:1039-1042.

13. Chalfie M: Neurosensory mechanotransduction. Nat Rev Mol Cell Biol 2009, 10:44-52.

14. Treinin $M$, Chalfie $M$ : A mutated acetylcholine receptor subunit causes neuronal degeneration in C. elegans. Neuron 1995, 14:871-877.

15. Halevi S, McKay J, Palfreyman M, Yassin L, Eshel M, Jorgensen E, Treinin M: The $C$. elegans ric- 3 gene is required for maturation of nicotinic acetylcholine receptors. EMBO J 2002, 21:1012-1020.

16. Treinin M, Gillo B, Liebman L, Chalfie M: Two functionally dependent acetylcholine subunits are encoded in a single Caenorhabditis elegans operon. Proc Natl Acad Sci USA 1998, 95:15492-15495.

17. Oren-Suissa M, Hall DH, Treinin M, Shemer G, Podbilewicz B: The fusogen EFF-1 controls sculpting of mechanosensory dendrites. Science 2010, 328:1285-1288.

18. Smith CJ, Watson JD, Spencer WC, O'Brien T, Cha B, Albeg A, Treinin M, Miller DM: Time-lapse imaging and cell-specific expression profiling reveal dynamic branching and molecular determinants of a multi-dendritic nociceptor in C. elegans. Dev Biol 2010, 345:18-33.

19. Husson SJ, Costa WS, Wabnig S, Stirman JN, Watson JD, Spencer WC, Akerboom J, Looger LL, Treinin M, Miller DM, Lu H, Gottschalk A: Optogenetic analysis of a nociceptor neuron and network reveals ion channels acting downstream of primary sensors. Curr Biol 2012 22:743-752

20. Li W, Feng Z, Sternberg PW, Shawn Xu XZ: A C. elegans stretch receptor neuron revealed by a mechanosensitive TRP channel homologue. Nature 2006, 440:684-687.

21. White J, Southgate E, Thomson J, Brenner S: The structure of the nervous system of the nematode Caenorhabditis elegans. Philos Trans R Soc Lond B Biol Sci 1986, 314:1-340.
22. Van Buskirk C, Sternberg PW: Epidermal growth factor signaling induces behavioral quiescence in Caenorhabditis elegans. Nat Neurosci 2007, 10:1300-1307

23. Mulcahy B, lent B: Meeting report: 2010 Caenorhabditis elegans neurobiology meeting, University of Wisconsin, USA. In Invert Neurosci 2010, 10:53-61.

24. Tian L, Hires SA, Mao T, Huber D, Chiappe ME, Chalasani SH, Petreanu L, Akerboom J, McKinney SA, Schreiter ER, Bargmann Cl, Jayaraman V, Svoboda K, Looger LL: Imaging neural activity in worms, flies and mice with improved GCaMP calcium indicators. Nature methods 2009, 6:875-881

25. Chalfie M, Sulston JE, White JG, Southgate E, Thomson JN, Brenner S: The neural circuit for touch sensitivity in Caenorhabditis elegans. J Neurosci 1985, 5:956-964.

26. Popovici C, Isnardon D, Birnbaum D, Roubin R: Caenorhabditis elegans receptors related to mammalian vascular endothelial growth factor receptors are expressed in neural cells. Neurosci Lett 2002, 329:116-120.

27. Wicks SR, Rankin CH: Integration of mechanosensory stimuli in Caenorhabditis elegans. J Neurosci 1995, 15:2434-2444.

28. Zheng Y, Brockie PJ, Mellem JE, Madsen DM, Maricq AV: Neuronal control of locomotion in C. elegans is modified by a dominant mutation in the GLR-1 ionotropic glutamate receptor. Neuron 1999, 24:347-361.

29. Tsalik EL, Hobert O: Functional mapping of neurons that control locomotory behavior in Caenorhabditis elegans. J Neurobio/ 2003, 56:178-197.

30. Gray J, Hill J, Bargmann C: A circuit for navigation in Caenorhabditis elegans. Proc Natl Acad Sci 2005, 127:3361-3371.

31. Faumont S, Rondeau G, Thiele TR, Lawton KJ, McCormick KE, Sottile M Griesbeck O, Heckscher ES, Roberts WM, Doe CQ, Lockery SR: An imagefree opto-mechanical system for creating virtual environments and imaging neuronal activity in freely moving Caenorhabditis elegans. PLOS ONE 2011, 6:e24666.

32. Li W, Kang L, Piggott BJ, Feng Z, Xu XZS: The neural circuits and sensory channels mediating harsh touch sensation in Caenorhabditis elegans. Nat Commun 2011, 2:315.

33. Piggott BJ, Liu J, Feng Z, Wescott SA, Xu XZS: The neural circuits and synaptic mechanisms underlying motor initiation in C. Elegans. Cell 2011, 147:922-933.

34. Kawano T, Po MD, Gao S, Leung G, Ryu WS, Zhen M: An imbalancing act: gap junctions reduce the backward motor circuit activity to bias $C$. Elegans for forward locomotion. Neuron 2011, 72:572-586.

35. Sawin ER, Ranganathan R, Horvitz HR: C. elegans locomotory rate is modulated by the environment through a dopaminergic pathway and by experience through a serotonergic pathway. Neuron 2000, 26:619-631.

36. Kindt KS, Viswanath V, Macpherson L, Quast K, Hu H, Patapoutian A, Schafer WR: Caenorhabditis elegans TRPA-1 functions in mechanosensation. Nat Neurosci 2007, 10:568-577.

37. Cohen E, Yemini E, Schafer W, Feitelson DG, Treinin M: Locomotion analysis identifies roles of mechanosensory neurons in governing locomotion dynamics of C. elegans. J Exp Biol 2012, 215:3639-3648.

38. Zhang M, Chung SH, Fang-Yen C, Craig C, Kerr RA, Suzuki H, Samuel ADT, Mazur E, Schafer WR: A self-regulating feed-forward circuit controlling C. elegans egg-laying behavior. Curr Biol 2008, 18:1445-1455.

39. Pujol N, Torregrossa P, Ewbank J, Brunet J: The homeodomain protein CePHOX2/CEH-17 controls antero-posterior axonal growth in C. elegans. Development 2000

40. Van Buskirk C, Sternberg PW: Paired and LIM class homeodomain proteins coordinate differentiation of the $\mathrm{C}$. elegans ALA neuron. Development 2010, 137:2065-2074

41. Arnadóttir J, O'Hagan R, Chen Y, Goodman MB, Chalfie M: The DEG/ENaC protein MEC-10 regulates the transduction channel complex in Caenorhabditis elegans touch receptor neurons. J Neurosci 2011 31:12695-12704

42. Maruyama IN, Brenner S: A phorbol ester/diacylglycerol-binding protein encoded by the unc-13 gene of Caenorhabditis elegans. Proc Natl Acad Sci USA 1991, 88:5729-5733.

43. Richmond JE, Davis WS, Jorgensen EM: UNC-13 is required for synaptic vesicle fusion in C. elegans. Nat Neurosci 1999, 2:959-964.

44. Kaplan JM, Horvitz HR: A dual mechanosensory and chemosensory neuron in Caenorhabditis elegans. Proc Natl Acad Sci USA 1993, 90:2227-2231. 
45. Hart AC, Sims S, Kaplan JM: Synaptic code for sensory modalities revealed by C. elegans GLR-1 glutamate receptor. Nature 1995, 378:82-85.

46. Hilliard MA, Apicella AJ, Kerr R, Suzuki H, Bazzicalupo P, Schafer WR: In vivo imaging of $\mathrm{C}$. elegans $\mathrm{ASH}$ neurons: cellular response and adaptation to chemical repellents. EMBO J 2005, 24:63-72.

47. Geffeney SL, Cueva JG, Glauser DA, Doll JC, Lee TH-C, Montoya M, Karania S, Garakani AM, Pruitt BL, Goodman MB: DEG/ENaC but not TRP channels are the major mechanoelectrical transduction channels in a $\mathrm{C}$. elegans nociceptor. Neuron 2011, 71:845-857.

48. Troemel ER, Chou JH, Dwyer ND, Colbert HA, Bargmann Cl: Divergent seven transmembrane receptors are candidate chemosensory receptors in C. elegans. Cell 1995, 83:207-218.

49. Sambongi Y, Nagae T, Liu Y, Yoshimizu T, Takeda K, Wada Y, Futai M: Sensing of cadmium and copper ions by externally exposed ADL, ASE, and ASH neurons elicits avoidance response in Caenorhabditis elegans. Neuroreport 1999, 10:753-757

50. Ward A, Liu J, Feng Z, Xu XZS: Light-sensitive neurons and channels mediate phototaxis in C. elegans. Nat Neurosci 2008, 11:916-922.

51. Jayanthi LD, Apparsundaram S, Malone MD, Ward E, Miller DM, Eppler M, Blakely RD: The Caenorhabditis elegans gene T23G5.5 encodes an antidepressant- and cocaine-sensitive dopamine transporter. Mol Pharmacol 1998, 54:601-609.

52. McCarroll SA, Li H, Bargmann Cl: Identification of transcriptional regulatory elements in chemosensory receptor genes by probabilistic segmentation. Curr Biol 2005, 15:347-352.

53. Schiavo G, Benfenati F, Poulain B, Rossetto O, Polverino de Laureto P, DasGupta BR, Montecucco C: Tetanus and botulinum-B neurotoxins block neurotransmitter release by proteolytic cleavage of synaptobrevin. Nature 1992, 359:832-835.

54. Macosko EZ, Pokala N, Feinberg EH, Chalasani SH, Butcher RA, Clardy J, Bargmann Cl: A hub-and-spoke circuit drives pheromone attraction and social behaviour in C. elegans. Nature 2009, 458:1171-1175.

55. Brenner S: The genetics of Caenorhabditis elegans. Genetics 1974, 77:71-94.

56. Chao MY, Komatsu H, Fukuto HS, Dionne HM, Hart AC: Feeding status and serotonin rapidly and reversibly modulate a Caenorhabditis elegans chemosensory circuit. Proc Natl Acad Sci USA 2004, 101:15512-15517.

57. Way JC, Chalfie M: The mec-3 gene of Caenorhabditis elegans requires its own product for maintained expression and is expressed in three neuronal cell types. Genes Dev 1989, 3:1823-1833.

58. Trent C, Tsuing N, Horvitz HR: Egg-laying defective mutants of the nematode Caenorhabditis elegans. Genetics 1983, 104:619-647.

59. Speese S, Petrie M, Schuske K, Ailion M, Ann K, Iwasaki K, Jorgensen EM, Martin TFJ: UNC-31 (CAPS) is required for dense-core vesicle but not synaptic vesicle exocytosis in Caenorhabditis elegans. J Neurosci 2007, 27:6150-6162

60. Chalfie M, Sulston J: Developmental genetics of the mechanosensory neurons of Caenorhabditis elegans. Dev Biol 1981, 82:358-370.

61. Maxwell I, Chung S, Mazur E: ScienceDirect.com - medical laser application - nanoprocessing of subcellular targets using femtosecond laser pulses. Med Laser Appl 2005, 20:193-200.

62. Chung SH, Mazur E: Surgical applications of femtosecond lasers. J Biophotonics 2009, 2:557-572

63. Yanik MF, Cinar H, Chisholm A, Jin Y, Ben-Yakar A: Neurosurgery functional regeneration after laser axotomy. Nature 2004, 432:822

64. Xu M, Jarrell TA, Wang Y, Cook SJ, Hall DH, Emmons SW: Computer assisted assembly of connectomes from electron micrographs: application to Caenorhabditis elegans. PLOS ONE 2013, 8:e54050.

65. Chalasani SH, Chronis N, Tsunozaki M, Gray JM, Ramot D, Goodman MB, Bargmann Cl: Dissecting a circuit for olfactory behaviour in Caenorhabditis elegans. Nature 2007, 450:63-70

66. Tsunozaki M, Chalasani SH, Bargmann Cl: A behavioral switch: CGMP and PKC signaling in olfactory neurons reverses odor preference in C. elegans. Neuron 2008, 59:959-971.

67. Suzuki H, Thiele TR, Faumont S, Ezcurra M, Lockery SR, Schafer WR: Functional asymmetry in Caenorhabditis elegans taste neurons and its computational role in chemotaxis. Nature 2008, 454:114-117.

68. Kuhara A, Okumura M, Kimata T, Tanizawa Y, Takano R, Kimura KD, Inada H, Matsumoto K, Mori I: Temperature sensing by an olfactory neuron in a circuit controlling behavior of C. elegans. Science 2008, 320:803-807.
69. Biron D, Wasserman S, Thomas JH, Samuel ADT, Sengupta P: An olfactory neuron responds stochastically to temperature and modulates Caenorhabditis elegans thermotactic behavior. Proc Natl Acad Sci USA 2008, 105:11002-11007.

70. Zimmer M, Gray JM, Pokala N, Chang AJ, Karow DS, Marletta MA, Hudson ML, Morton DB, Chronis N, Bargmann Cl: Neurons detect increases and decreases in oxygen levels using distinct guanylate cyclases. Neuron 2009, 61:865-879.

71. Sarria I, Gu J: Menthol response and adaptation in nociceptive-like and nonnociceptive-like neurons: role of protein kinases. Mol Pain 2010, 6:47.

72. Wolf JA, Stys PK, Lusardi T, Meaney D, Smith DH: Traumatic axonal injury induces calcium influx modulated by tetrodotoxin-sensitive sodium channels. J Neurosci 2001, 21:1923-1930.

73. Kim E, Sun L, Gabel CV, Fang-Yen C: Long-term imaging of Caenorhabditis elegans using nanoparticle-mediated immobilization. PLOS ONE 2013, 8:e53419.

74. Nelson MD, Raizen DM: A sleep state during C. elegans development. Curr Opin Neurobiol 2013

75. Zahn TR, Macmorris MA, Dong W, Day R, Hutton JC: IDA-1, a Caenorhabditis elegans homolog of the diabetic autoantigens IA-2 and phogrin, is expressed in peptidergic neurons in the worm. J Comp Neurol 2001, 429:127-143.

76. Cai T, Fukushige T, Notkins AL, Krause M: Insulinoma-associated protein IA-2, a vesicle transmembrane protein, genetically interacts with UNC-31/CAPS and affects neurosecretion in Caenorhabditis elegans. J Neurosci 2004, 24:3115-3124.

77. Plummer MR, Kirk MD: Premotor neurons B51 and B52 in the buccal ganglia of Aplysia californica: synaptic connections, effects on ongoing motor rhythms, and peptide modulation. J Neurophysiol 1990, 63:539-558.

78. Evans CG, Cropper EC: Proprioceptive input to feeding motor programs in Aplysia. J Neurosci 1998, 18:8016-8031.

79. Edelstein A, Amodaj N, Hoover K, Vale R, Stuurman N: Computer control of microscopes using $\mu$ Manager. Curr Protoc Mol Biol 2010, 14:14.20.1. 14.20 .17$.

doi:10.1186/1471-2202-14-156

Cite this article as: Sanders et al:: The Caenorhabditis elegans interneuron ALA is (also) a high-threshold mechanosensor. BMC Neuroscience 2013 14:156.

\section{Submit your next manuscript to BioMed Central and take full advantage of:}

- Convenient online submission

- Thorough peer review

- No space constraints or color figure charges

- Immediate publication on acceptance

- Inclusion in PubMed, CAS, Scopus and Google Scholar

- Research which is freely available for redistribution 